
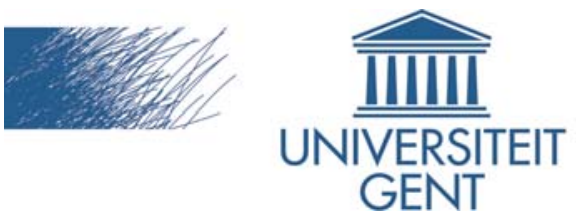

biblio.ugent.be

The UGent Institutional Repository is the electronic archiving and dissemination platform for all UGent research publications. Ghent University has implemented a mandate stipulating that all academic publications of UGent researchers should be deposited and archived in this repository. Except for items where current copyright restrictions apply, these papers are available in Open Access.

This item is the archived peer-reviewed author-version of: Quantitative risk assessment via uncertainly analysis in combination with error propagation for the determination of the dynamic Design Space of the primary drying step during freeze-drying

Authors: Van Bockstal P.J., Mortier S., Corver J., Nopens I.? Gernaey K.V., De Beer T.

In: European Journal of Pharmaceutics and Biopharmaceutics 2017, 121: 32-41

To refer to or to cite this work, please use the citation to the published version:

Van Van Bockstal P.J., Mortier S., Corver J., Nopens I.? Gernaey K.V., De Beer T. (2017)

Quantitative risk assessment via uncertainly analysis in combination with error propagation for the determination of the dynamic Design Space of the primary drying step during freezedrying

European Journal of Pharmaceutics and Biopharmaceutics 121: 32-41

DOI: 10.1016/j.ejpb.2017.08.015 


\title{
Quantitative risk assessment via uncertainty analysis in combination with error propagation for the determination of the dynamic Design Space of the primary drying step during freeze-drying
}

\author{
Pieter-Jan Van Bockstal ${ }^{\mathrm{a}}$, Séverine Thérèse F.C. Mortier ${ }^{\mathrm{a}, \mathrm{b}}$, Jos Corver ${ }^{\mathrm{a}}$, Ingmar Nopens ${ }^{\mathrm{b}}$, Krist V. \\ Gernaey $^{\mathrm{c}}$, Thomas De Beer $\mathrm{T}^{\mathrm{a}, 1,2, *}$ \\ ${ }^{a}$ Laboratory of Pharmaceutical Process Analytical Technology, Department of Pharmaceutical Analysis, Faculty of \\ Pharmaceutical Sciences, Ghent University, Ottergemsesteenweg 460, 9000 Ghent, Belgium \\ ${ }^{b}$ BIOMATH, Department of Mathematical Modelling, Statistics and Bioinformatics, Faculty of Bioscience Engineering, \\ Ghent University, Coupure Links 653, 9000 Ghent, Belgium \\ ${ }^{c}$ Process and Systems Engineering Center (PROSYS), Department of Chemical and Biochemical Engineering, Technical \\ University of Denmark, Building 229, 2800 Kgs. Lyngby, Denmark
}

\begin{abstract}
Traditional pharmaceutical freeze-drying is an inefficient batch process often applied to improve the stability of biopharmaceutical drug products. The freeze-drying process is regulated by the (dynamic) settings of the adaptable process parameters shelf temperature $T_{s}$ and chamber pressure $P_{c}$. Mechanistic modelling of the primary drying step allows the computation of the optimal combination of $T_{s}$ and $P_{c}$ in function of the primary drying time. In this study, an uncertainty analysis was performed on the mechanistic primary drying model to construct the dynamic Design Space for the primary drying step of a freeze-drying process, allowing to quantitatively estimate and control the risk of cake collapse (i.e., the Risk of Failure (RoF). The propagation of the error on the estimation of the thickness of the dried layer $L_{d r i e d}$ as function of primary drying time was included in the uncertainty analysis. The constructed dynamic Design Space and the predicted primary drying endpoint were experimentally verified for different RoF acceptance levels $(1 \%, 25 \%, 50 \%$ and $99 \% \mathrm{RoF}$, defined as the chance of macroscopic cake collapse in one or more vials. An acceptable cake structure was only obtained for the verification runs with a preset RoF of $1 \%$ and $25 \%$. The run with the nominal values for the input variables $(\mathrm{RoF}$ of $50 \%)$ led to collapse in a significant number of vials. For each RoF acceptance level, the experimentally determined primary drying endpoint was situated below the computed endpoint, with a certainty of $99 \%$, ensuring sublimation was finished before secondary drying was started. The uncertainty on the model input parameters demonstrates the need of the uncertainty analysis for the determination of the dynamic Design Space to quantitatively estimate the risk of batch rejection due to cake collapse.
\end{abstract}

Keywords: Freeze-drying, Mathematical modelling, Dynamic Design Space, Quantitative risk assessment, Error propagation, Risk of failure control

\section{Introduction}

Pharmaceutical freeze-drying is a process often used to improve the stability of biopharmaceutical drug products with a limited stability when formulated as an aqueous solution [1]. Traditionally, freeze-drying is

\footnotetext{
* Corresponding author

Email addresses: pieterjan.vanbockstal@ugent.be (Pieter-Jan Van Bockstal), severine.mortier@ugent.be (Séverine Thérèse F.C. Mortier), jos.corver@RheaVita.nl (Jos Corver), ingmar.nopens@ugent.be (Ingmar Nopens), KVG@kt.dtu.dk (Krist V. Gernaey), thomas.debeer@ugent.be (Thomas De Beer )

${ }^{1}$ Phone number: +32(0)9 2648097

${ }^{2}$ Fax number: +32(0)92228236
} 
a batch-wise process during which all vials of each batch are processed through a sequence of consecutive steps (i.e. freezing, primary and secondary drying) until the dried end product is obtained [2]. Glass vials containing the aqueous drug formulation (i.e. unit doses) are loaded onto temperature-controlled shelves in the drying chamber. At the start of the freezing stage, these shelves are gradually cooled until approximately $-45^{\circ} \mathrm{C}$, depending on the formulation characteristics. The temperature of the aqueous drug formulation decreases until ice nucleation takes place and part of the water is converted into ice. While ice crystal growth proceeds upon further cooling, the solutes gradually concentrate between these crystals (freeze-concentration) [3]. At the end of the freezing step, the shelf temperature reaches a value well below the eutectic temperature $T_{e}$ or the glass transition temperature of the maximum freeze-concentrated formulation $T_{g}^{\prime}$ for crystalline or amorphous products, respectively, resulting in complete solidification of the product. To allow full crystallization of crystalline components or to enhance the batch uniformity of the ice crystal size, an annealing step can be added to the freezing stage 46 . Here, the shelf temperature at the end of the freezing step is raised to a temperature above $T_{g}^{\prime}$, but below $T_{e}$. This shelf temperature is maintained for several hours before re-establishing the final freezing temperature, below $T_{g}^{\prime}$. The primary drying step is initiated by lowering the chamber pressure, conventionally to a value between 5 and $30 \mathrm{~Pa}$. When the desired pressure is reached, the shelf temperature is raised to promote ice sublimation. The sublimation front, i.e. the interface separating ice-free product from the ice crystal matrix, moves gradually downward with the progress of primary drying. When ice sublimation is completely finished, the shelf temperature is further raised during the secondary drying step supporting the desorption of unfrozen water in the amorphous matrix and/or the removal of hydrate water in crystalline material until the desired residual moisture content of the end product is obtained.

One of the Critical Quality Attributes (CQAs) of lyophilized biopharmaceutical products, defined as product properties or characteristics that should meet defined standards to ensure the desired quality (ICH Q8), is the cake appearance [7. Loss of cake structure (collapse) should be avoided throughout the lyophilization process to ensure reconstitution of the drug product within an appropriate timing and for aesthetic purposes [1. For this reason, the product temperature at the sublimation front $T_{i}$ should be maintained below the critical product temperature $T_{i, c r i t}$ during the entire primary drying step. $T_{i, c r i t}$ is characteristic for each formulation and identified as the collapse temperature $T_{c}$ or $T_{e}$ for amorphous and crystalline products, respectively. In general, $T_{c}$ lies a few degrees above $T_{g}^{\prime}$ because the viscosity near $T_{g}^{\prime}$ is sufficiently high to limit viscous flow 3]. During secondary drying, the product temperature should be kept below the glass transition temperature of the dried product $T_{g}$ to avoid molecular motion and loss of cake structure. The value of $T_{g}$ is much higher compared to $T_{g}^{\prime}$ of the freeze-concentrate and is highly influenced by the residual moisture content, due to the plasticizing effect of water 1]. In general, the shelf temperature ramp during the transition of the primary to secondary drying step is rather conservative, due to the potential high residual moisture content immediately after primary drying. However, several approaches have been developed to optimize this transition phase [8, 9].

The freeze-drying process is characterized by two adaptable process variables, i.e. the shelf fluid inlet temperature $T_{s}$ and chamber pressure $P_{c}$. Combinations of both parameters are set in function of time, specific for each process step. During primary drying, the settings of $T_{s}$ and $P_{c}$ should result in a value for $T_{i}$ associated with maximum sublimation efficiency, while maintaining an appropriate cake structure. Mechanistic models based on the fundamental understanding of the underlying physical mechanisms of the sublimation process, allow the determination of the optimal combination of $T_{s}$ and $P_{c}$ during primary drying and their calibration for a formulation to be freeze-dried [10, 11. The development of these mechanistic models only requires a limited amount of experiments, compared to data-driven empirical models associated with a high experimental load. Mechanistic modelling assists in cycle development and optimization by computing $T_{i}$ for different combinations of $T_{s}$ and $P_{c}$, eventually leading to the construction of the Design Space for primary drying [12 14]. The Design Space is defined as the multidimensional combination and interaction of input variables and process parameters leading to an acceptable product quality with a controlled probability (ICH Q8) [7. For primary drying, the Design space is demarcated by the equipment limitations, the sublimation efficiency and the specific CQA for the lyophilized biopharmaceutical product, in this case the dried cake appearance [15-17]. All combinations of $T_{s}$ and $P_{c}$ within these limits lead to a lyophilized product with an appropriate cake appearance that is achieved within an acceptable time-frame. Model input variables can 
change with the progress of primary drying, such as the thickness of the dried product layer $L_{\text {dried }}$. Upon an increase in $L_{d r i e d}$, the path of the water vapour originating from the sublimation interface through the pores of the above dried product layer becomes longer, associated with an increase in the dried product mass transfer resistance $R_{p}$. Therefore, the heat transfer to the product should be gradually lowered, to avoid an increase in $T_{i}$. As a consequence, the optimal combination of $T_{s}$ and $P_{c}$ changes in time to maintain $T_{i}$ below $T_{i, c r i t}$, leading to a dynamic Design Space [12, 13].

The mechanistic models are a mathematical approximation of the sublimation process during the primary drying stage. Inherent to model development, a few assumptions and simplifications are included in these primary drying models (e.g. a planar sublimation front and steady-state system are assumed). In addition, the model input variables and process parameters are in some cases an estimation of the real value. Some input variables (i.e. $R_{p}$ and the vial heat transfer coefficient $K_{v}$ are quantified by one global value for the entire batch, while they can differ from vial to vial (inter-vial variability). For these reasons, the computational model predictions are associated with a degree of uncertainty, through which they can deviate from the actual (experimental) drying behaviour. By including this parameter uncertainty into the mechanistic primary drying model, the uncertainty on the model predictions can be determined leading to the quantitative estimation of the $\mathrm{RoF}$ (i.e. risk of cake collapse) for each combination of the adaptable process variables, $P_{c}$ and $T_{s}$, in the dynamic Design Space. Only a limited number of studies have been conducted focusing on quantitative risk assessment. The uncertainty of two input variables, $R_{p}$ and $K_{v}$, can be compensated by assuming a Gaussian distribution of the values around their mean [10, 18. Alternatively, the impact of the variation in $R_{p}$ and $K_{v}$ was assessed by determining the variance for these parameters and their influence on the primary drying time [19. In both approaches, only $R_{p}$ and $K_{v}$ were considered as uncertain parameters. In previous work, the use of a dedicated uncertainty analysis was presented, in which the uncertainty on each uncertain input variable and process parameter was estimated and quantified [13. However, $L_{d r i e d}$ is both an input and output variable of the mechanistic model. As the value of $L_{\text {dried }}$ is computed throughout the simulation, the uncertainty on this input parameter results from the other uncertain input variables and, in addition, the uncertainty increases with the drying progress due to the accumulation of error. In this paper, the aforementioned dedicated uncertainty analysis was refined and extended with a strategy to incorporate the accumulation of error into the primary drying model. The combination of the dedicated uncertainty analysis with the time dependent process variables results in the dynamic Design Space with the ultimate aim of quantifying and controlling the risk of cake collapse and reducing the risk of batch rejection, while maximizing the drying efficiency.

\section{Materials and methods}

\subsection{Freeze-drying model}

The mechanistic model describing the primary drying step of a pharmaceutical batch freeze-drying process is described in detail by Mortier et al. [13. The nominal values of the model input parameters are listed in table 1. The coefficients describing $K_{v}$ were experimentally determined, as explained in section 2.3 The coefficients describing $R_{p}$ for the model formulation were taken from literature [20]. The aqueous model formulation contained $30 \mathrm{mg} / \mathrm{mL}$ of sucrose (Sigma-Aldrich, Saint-Louis, MO, USA). $T_{i, c r i t}$ was assumed to be equal to $T_{q}^{\prime}$ of the model formulation, which was determined using Modulated Differential Scanning Calorimetry (MDSC) (Section 2.4). A planar sublimation interface was assumed for the model. Finally, the system was considered to be at steady-state, i.e. the transferred energy was only used for ice sublimation.

To limit the computational load, the grid for $P_{c}$ and $T_{s}$ for the grid-search was limited. As the optimal value for the chamber pressure is seldom increasing after reaching the vacuum conditions, the limits for $P_{c}$ were set at $[10 \mathrm{~Pa} 14 \mathrm{~Pa}]$ after the exponential pressure decrease at the start of primary drying [13]. The lower boundary was chosen based on the lower limit that can be reached by the equipment. The upper boundary was initially set based on expert knowledge. In case this upper boundary was reached during the grid-search, the upper limit was extended. The grid boundaries for $T_{s}$ changed during the process, and the range was based on the maximum temperature ramp that can be supported by the freeze-dryer, i.e. $1^{\circ} \mathrm{C} / \mathrm{min}$. The time step used for the calculations increased with the progress of primary drying. Consequently, the width 
Table 1: Nominal values of the parameters of the model describing the primary drying step

\begin{tabular}{c|c|c} 
Parameter & & Numerical value \\
\hline Inner radius of 10R vial & $r_{v, i}$ & $0.011 \mathrm{~m}$ \\
Outer radius of 10R vial & $r_{v, o}$ & $0.012 \mathrm{~m}$ \\
Radius of the vial neck & $r_{v, n}$ & $0.0063 \mathrm{~m}$ \\
Radius of the duct of the dryer & $r_{d}$ & $0.08 \mathrm{~m}$ \\
$K_{v}$-coefficient & $\alpha$ & $\left.11.18 \mathrm{~J} / \mathrm{m}^{2} \mathrm{sK}\right)$ \\
$K_{v}$-coefficient & $\beta$ & $1.435 \mathrm{~J} /\left(\mathrm{m}^{2} \mathrm{sKPa}\right)$ \\
$K_{v}$-coefficient & $\gamma$ & $0.040081 / \mathrm{Pa}$ \\
$R_{p}$-coefficient & $R_{p, 0}$ & $6.92610^{4} \mathrm{~m} / \mathrm{s}$ \\
$R_{p}$-coefficient & $A_{R p}$ & $9.12410^{6} 1 / \mathrm{s}$ \\
$R_{p}$-coefficient & $B_{R p}$ & $01 / \mathrm{m}$ \\
Filling volume & $T_{i, c r i t}$ & $-32.5^{\circ} \mathrm{C}$ \\
Density of ice & $V$ & $2.5 \mathrm{~mL}$ \\
Critical product temperature & $\rho_{i c e}$ & $919.4 \mathrm{~kg} / \mathrm{m}{ }^{3}$ \\
Volume fraction of ice & $\epsilon$ & 0.97 \\
Ratio of the specific heat for water & $k$ & 1.33 \\
Molecular weight of water & $M$ & $0.018015 \mathrm{~kg} / \mathrm{mol}$
\end{tabular}

of the range for $T_{s}$ changed accordingly throughout the calculations based on the value of $T_{s}$ for the previous time step and the maximum ramp of $T_{s}$, e.g., for a value of $-20^{\circ} \mathrm{C}$ for $T_{s}$ and a time step of 5 minutes, the range is $\left[-25^{\circ} \mathrm{C}-15^{\circ} \mathrm{C}\right]$.

The optimal process settings were determined on both vial and dryer-level. On vial-level, only choked flow at the vial neck is taken into account, as it is the most narrow point for the mass transfer, which is calculated using the following relation [17]:

$$
\dot{m}_{\text {sub,chok,vial }}=\frac{0.3 r_{v, n}^{2} \pi \sqrt{\frac{k T_{r} R}{M}} M}{R T_{r}} P_{c}
$$

with $\dot{m}_{\text {sub,chok,vial }}$ the maximum sublimation rate avoiding choked flow at vial level $(\mathrm{kg} / \mathrm{s}), r_{v, n}$ the equivalent radius corresponding with the most narrow lumen at the stopper placed upon the vial neck opening (m), $k$ the ratio of the specific heat for water (1.33), $T_{r}$ the temperature of the water vapour $(\mathrm{K}), R$ the gas constant $(8.3144621 \mathrm{~J} /(\mathrm{K} \mathrm{mol}))$ and $M$ the molecular weight of water $(\mathrm{kg} / \mathrm{mol})$. The choked flow at dryerlevel, where the valve in the duct connecting the drying chamber and the condenser is the limiting factor, is given by [17]:

$$
\dot{m}_{\text {sub,chok,dryer }}=\frac{0.3 r_{d}^{2} \pi \sqrt{\frac{k T_{r} R}{M}} M}{R T_{r}} \frac{P_{c}}{n_{\text {vials }}}
$$

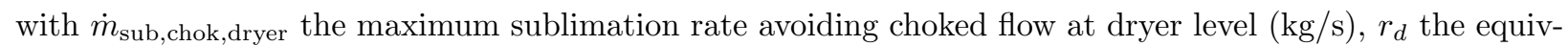
alent radius corresponding with the most narrow lumen at the valve connecting the drying chamber and condenser $(\mathrm{m})$ and $n_{\text {vials }}$ the theoretical maximum number of vials that can fit in the drying chamber of the freeze-dryer $(1,140)$. Here, $r_{d}$ is defined for the used equipment, equipped with a butterfly valve. For other types of equipment, $r_{d}$ can be defined as the radius of the duct connecting drying chamber and condenser itself.

\subsection{Uncertainty analysis}

The uncertainty analysis on the freeze-drying model leading to the dynamic Design Space was conducted using the sampling-based approach 21. For this method, the model is run for different combinations of input variables and process parameters, based on their uncertainty range. Next, the uncertainty on the model output is estimated from the predictions at these sample points. The input matrix containing 10,000 
samples (i.e. the sampling scheme) was constructed using the Sobol sampling technique [22]. The analysis was done for 8 factors that were considered to be uncertain. $T_{i, c r i t}$ was assumed to be equal to $T_{g}^{\prime}$ of the model formulation. In general, $T_{g}^{\prime}$ is a few degrees lower than $T_{c}$, the temperature at which collapse actually occurs. This should provide a sufficient safety margin to avoid cake collapse. For this reason, $T_{i, c r i t}$ was not considered as one of the uncertain factors. The uncertainty levels for each factor are given in table 2. For most factors, the uncertainty was not implemented as a percentage of the nominal value as the range is highly dependent on the magnitude of the factor. For these factors (i.e., $T_{s}, P_{c}, r_{v, i}$ and $r_{v, o}$ ), the uncertainty was defined by an absolute range, based on experimental data or data provided by the supplier (see further). $L_{\text {dried }}$ was calculated for each time step throughout the process, based on the other (uncertain) model input variables. Therefore, there is uncertainty about the exact value of this input variable due to the uncertainty of the initial conditions and the model parameters. In addition, the uncertainty on $L_{d r i e d}$ increases throughout the simulation due to the accumulation of error in the previous steps. The uncertainty level was determined by calculating the standard deviation on a fixed number of samples, i.e. 1,000 samples, by varying the values of the model parameters within their specified ranges. $K_{v}$ is described in function of $P_{c}$ via [13]:

$$
K_{v}=\alpha+\frac{\beta P_{c}}{1+\gamma P_{c}}
$$

with $\alpha\left(\mathrm{J} /\left(\mathrm{m}^{2} \mathrm{sK}\right), \beta\left(\mathrm{J} /\left(\mathrm{m}^{2} \mathrm{sKPa}\right)\right.\right.$ and $\gamma(1 / \mathrm{Pa})$ constants describing $K_{v}$ quantitatively in function of $P_{c}$ (Table 1). $R_{p}$ is described in function of $L_{\text {dried }}$ via [13]:

$$
R_{p}=R_{p, 0}+\frac{A_{R p} L_{d r i e d}}{1+B_{R p} L_{d r i e d}}
$$

with $R_{p, 0}(\mathrm{~m} / \mathrm{s}), A_{R p}(1 / \mathrm{s})$ and $B_{R p}(1 / \mathrm{m})$ constants describing $R_{p}$ quantitatively in function of $L_{d r i e d}$ (Table 1). The uncertainty on these coefficients is lumped by introducing an uncertainty on the entire parameter, i.e. $R_{p}$ and $K_{v}$. The uncertainty on the inner and outer radius of the vials, $r_{v, i}$ and $r_{v, o}$, is based on data provided by the supplier of the $10 \mathrm{R}$ vials (Schott, Müllheim, Germany). The filling volume $V$ was included in the analysis because of the inherent error during the filling stage. Depending on the location of the vial on the shelf, $T_{s}$ can be somewhat higher or lower than the set value. This error has been experimentally determined for both a constant value of $T_{s}$ and during a shelf temperature ramp. The uncertainty level for $T_{s}$ is based on these preliminary experiments. When the shelf fluid inlet temperature is kept constant, the uncertainty level on $T_{s}$ is fixed at $2.5^{\circ} \mathrm{C}$. During a shelf temperature ramp, it was revealed that $T_{s}$ reaches the set value with a delay due to thermal inertia of the shelves, leading to a broadening of the uncertainty range. When the shelf temperature is set to increase, the upper uncertainty level remains fixed at $2.5^{\circ} \mathrm{C}$, while the lower level linearly increases from $2.5^{\circ} \mathrm{C}$ until a maximum of $10^{\circ} \mathrm{C}$ at the same rate as the temperature ramp (Figure 1). The lower limit remains $10^{\circ} \mathrm{C}$ as long as $T_{s}$ increases, until the set value is reached and the uncertainty on $T_{s}$ gradually vanishes within 15 minutes. Vice versa, when the shelf temperature is set to decrease, the lower level of the uncertainty range remains constant while the upper level increases. The error on $P_{c}$ is based on the maximum allowed deviation of the feedback pressure control system.

\subsection{Determination of the vial heat transfer coefficient}

$K_{v}$ was determined via a gravimetric method [23. A total of 100 type I glass vials, each filled with $2.5 \mathrm{~mL}$ of deionized water, were placed on the precooled shelves $\left(3^{\circ} \mathrm{C}\right)$ in the drying chamber. The shelves were further cooled until $-45^{\circ} \mathrm{C}$ at a rate of $1^{\circ} \mathrm{C} / \mathrm{min}$. This temperature was maintained for 1 hour. Next, the temperature was linearly increased until $-20^{\circ} \mathrm{C}$ in 25 minutes, after which the pressure was lowered until the desired value. After 6 hours of primary drying, ice sublimation was interrupted by aerating the drying chamber with dry nitrogen and subsequently closing the vials. Each individual vial was weighed before and after primary drying. The value of $K_{v}$ changes in function of $P_{c}$, therefore, this cycle was repeated at six different pressure levels: 5, 10, 15, 20, 25 and $30 \mathrm{~Pa}$. Type-K thermocouples (Conrad Electronic, Hirschau, 
Table 2: Uncertainty level for the input factors of the uncertainty analysis

\begin{tabular}{c|c|c} 
Factor & Uncertainty level & Reason of inclusion in uncertainty analysis \\
\hline$L_{\text {dried }}$ & - & Error propagation \\
$R_{p}$ & $20 \%$ & Based on experimental data \\
$K_{v}$ & $5 \%$ & Based on experimental data \\
$r_{v, i}$ & $10^{-4} \mathrm{~m}$ & Process variable with inherent error \\
$r_{v, o}$ & $10^{-4} \mathrm{~m}$ & Process variable with inherent error \\
$V$ & $1 \%$ & Process variable with inherent error \\
$T_{s}$ & $2.5-10^{\circ} \mathrm{C}$ & Based on experimental data \\
$P_{c}$ & $1 \mathrm{~Pa}$ & Process variable with inherent error
\end{tabular}

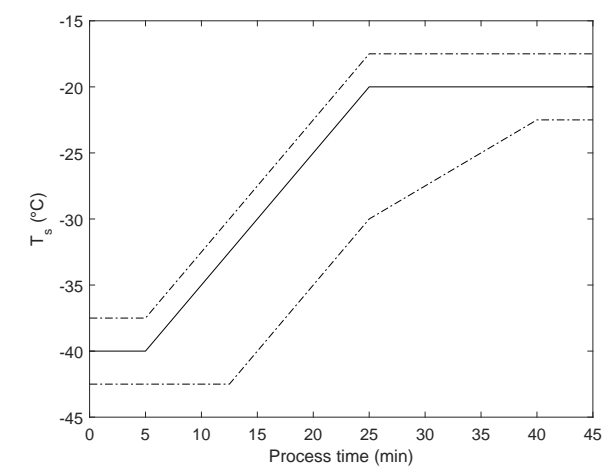

Figure 1: Illustration of the uncertainty on $T_{s}$ during temperature ramp: theoretical (set) value of $T_{s}$ (solid line) with uncertainty levels (dash dotted line) in function of process time

Germany) were placed inside four different vials, which were randomly distributed at the border and the center of the batch, against the bottom in the center of the vial, to monitor the ice temperature during primary drying [24]. $K_{v}$ was calculated at each pressure level according to equation 5 [12]:

$$
K_{v}=\frac{\Delta m \Delta H_{\text {sub }}}{\Delta t\left(T_{s}-T_{b}\right) A_{v} M}
$$

with $\Delta m$ the mass of sublimated ice $(\mathrm{kg}), \Delta H_{\text {sub }}$ the latent sublimation heat of ice $(\mathrm{J} / \mathrm{mol}), \Delta t$ the primary drying time interval $(\mathrm{s}), T_{s}$ the shelf fluid inlet temperature $(\mathrm{K}), T_{b}$ the ice temperature at the bottom of the vial $(\mathrm{K})$ and $A_{v}$ the outer vial area $\left(\mathrm{m}^{2}\right)$. For each pressure level, equation 3 was fitted to the value of $K_{v}$ via nonlinear regression to obtain these coefficients. Because of the difference in radiation energy coming from the surrounding walls, discrimination was made between vials situated in the center and at the edge of the batch. The first group consisted of vials which were at all four sides surrounded by other vials (center vials). The second group consisted of vials situated at the edge of the shelf, with at least one side directed towards the door or the walls of the drying chamber (edge vials). The parameters $\alpha, \beta$ and $\gamma$ describing $K_{v}$ in function of $P_{c}$, were determined separately for both groups. This implies that $K_{v}$ combines both the radiative and conductive energy transfer. The total energy transfer to the edge vials is higher compared to the center vials (Section 3.1), which is associated with a higher risk of cake collapse for the vials situated at the border of the batch. Because they are the limiting factor, $\alpha, \beta$ and $\gamma$ for the edge vials were selected as input variables for the freeze-drying model to compute the dynamic Design Space.

\subsection{Determination of the critical product temperature}

$T_{i, \text { crit }}$ of the model formulation was determined via MDSC using a differential scanning calorimeter Q2000 (TA instruments, Zellik, Belgium). Hermetically sealed aluminum pans (TA instruments, Zellik, Belgium) 
were filled with approximately $12 \mathrm{mg}$ of the model formulation, containing $30 \mathrm{mg} / \mathrm{mL}$ sucrose. The DSC cell was constantly purged with dry nitrogen at a flow rate of $50 \mathrm{~mL} / \mathrm{min}$. Initially, the sample was rapidly cooled until $-90^{\circ} \mathrm{C}$. This temperature was maintained for 5 minutes. Subsequently the temperature was linearly increased until $0{ }^{\circ} \mathrm{C}$ at a heating rate of $2{ }^{\circ} \mathrm{C} / \mathrm{min}$. The modulation amplitude and period were set at $0.212{ }^{\circ} \mathrm{C}$ and 40 seconds, respectively. The analysis was performed in triplicate. The thermograms were analyzed with TA Instruments Universal Analysis 2000 version 4.7A (TA Instruments, Zellik, Belgium).

\subsection{Experimental verification of the dynamic Design Space}

Four experimental runs were conducted to verify the dynamic Design Space for the model formulation under study. The settings of the adaptable process parameters $T_{s}$ and $P_{c}$ were computed for different RoF acceptance levels (1\%, 25\%, 50\% and 99\%). The RoF acceptance level is defined as the chance of batch rejection due to macroscopic cake collapse in one or more vials. The experiments were conducted in a laboratory scale freeze-dryer Lyobeta 25 (Telstar, Terrassa, Spain) equipped with four shelves, each with a heatable area of $0.16 \mathrm{~m}^{2}$, in the vacuum-tight drying chamber with a volume of $0.2 \mathrm{~m}^{3}$. The system contains a condenser with $35 \mathrm{~kg}$ of ice capacity and a vacuum pump to evacuate the drying chamber. Chamber pressure control was performed via a Baratron Type 626A capacitance manometer (MKS Instruments, Andover, MA, USA). Comparative pressure measurements were conducted using a Pirani Type PSG-502-S thermal conductivity gauge (Inficon, Bad Ragaz, Switzerland). Temperature control of shelf and condenser was based on Pt100 thermal sensors (WIKA Instruments, Klingenberg, Germany) measurements.

The freezing step for each verification run was identical to the freezing procedure used to determine $R_{p}$ of the model formulation [20]. A total of 100 glass vials, each filled with $2.5 \mathrm{~mL}$ of the model formulation, was placed on the precooled shelves $\left(3^{\circ} \mathrm{C}\right)$ in the drying chamber. The shelves were chilled at a rate of $1{ }^{\circ} \mathrm{C} / \mathrm{min}$ until $-45^{\circ} \mathrm{C}$. This temperature was maintained for 1 hour. An annealing step was included in the freezing procedure, during which $T_{s}$ was increased in 25 minutes until $-20^{\circ} \mathrm{C}$ and kept at this value for 2 hours. After re-establishing the final freezing temperature of $-45^{\circ} \mathrm{C}$ at a rate of $1{ }^{\circ} \mathrm{C} / \mathrm{min}, T_{s}$ was kept for another hour at this value, before the condenser was cooled and the vacuum pump was activated. The primary drying settings were characteristic for each verification run and the corresponding RoF acceptance level. When the endpoint of primary drying was detected, secondary drying was conducted at $20^{\circ} \mathrm{C}$, after ramping the temperature at $1{ }^{\circ} \mathrm{C} / \mathrm{min}$ starting from the final $T_{s}$ at the end of primary drying. The value of $P_{c}$ during secondary drying was kept equal to the one during primary drying. At the end of each run, the vials were stoppered under a controlled nitrogen atmosphere.

The dynamic Design Space was computed based on the coefficients describing $K_{v}$ for the edge vials (Table 1). As compensation for the slower sublimation rate in the center vials (Section 3.1), the primary drying phase of each verification run was prolonged by maintaining the final optimal combination of $T_{s}$ and $P_{c}$ for a few additional hours, on top of the predicted dynamic primary drying trajectory for the edge vials. The total drying time for the complete batch, including the center vials, was predicted based on the coefficients describing $K_{v}$ for these center vials. The computed optimal dynamic settings of $T_{s}$ and $P_{c}$ were used as input for the primary drying model to calculate the sublimation rate $\dot{m}_{\text {sub }}$ using equation 6 [13]:

$$
\dot{m}_{\mathrm{sub}}=A_{p} \frac{\left(P_{w, i}-P_{w, c}\right)}{R_{p}}
$$

where $A_{p}$ is the product area available for sublimation $\left(\mathrm{m}^{2}\right), P_{w, i}$ the vapour pressure of ice at the sublimation interface $(\mathrm{Pa}), P_{w, c}$ the partial pressure of water in the drying chamber $(\mathrm{Pa})$. Here, $P_{w, c}$ is assumed to be equal to $P_{c}$, as during primary drying the gas composition in the drying chamber consists almost entirely of water vapour [12. The nominal values of the input parameters (Table 1) and uncertainty levels (Table 2) required for the calculation of the total primary drying time were equal to the ones used for the calculation of the dynamic Design Space, with the coefficients describing $K_{v}$ for the center vials instead of the edge vials. The propagation of errors on $L_{\text {dried }}$ was not included for the computation of the total drying time, as the primary drying endpoint was based upon complete sublimation of the ice mass instead of correlating $L_{\text {dried }}$ with the total layer thickness to avoid an overestimation of the drying time. For each verification run, the primary drying endpoint was computed with a certainty of $99 \%$ based on the uncertainty level of 
each parameter, as illustrated in figure 2, ensuring the batch is completely dry.

The end of primary drying was detected based on the ratio of the Pirani thermal conductivity gauge and

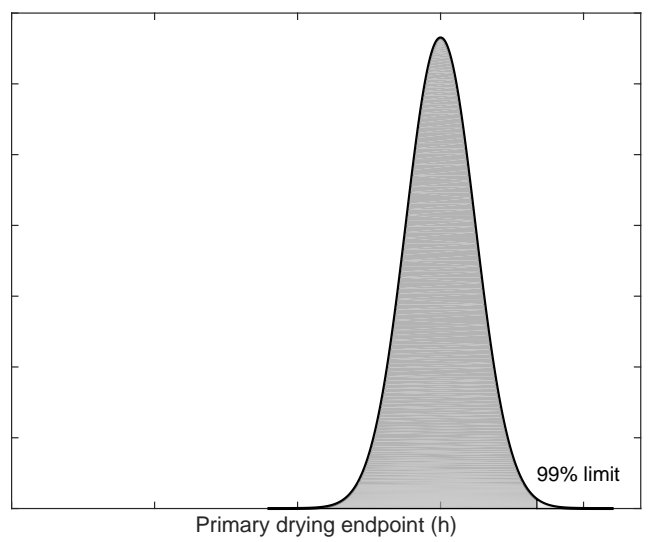

Figure 2: Illustration of the computation of the primary drying endpoint with a certainty of $99 \%$

the Baratron capacitance manometer. The capacitance manometer measures the absolute pressure, while the relative pressure measurement of the thermal conductivity gauge changes with the gas composition in the drying chamber. The gas composition changes from mainly water vapour during primary drying to mostly nitrogen towards the end of primary drying, when ice sublimation is complete. Primary drying was considered finished at the midpoint of the drop in Pirani pressure 25]. As this comparative pressure measurement method monitors the entire batch, the endpoint is only reached when the last vials of the batch are dry.

$T_{i}$ was constantly monitored via thermocouples in contact with the bottom of the vials, to evaluate whether $T_{i, c r i t}$ was exceeded during primary drying or not. Vials situated in the center and at the edge of the batch were monitored, which allowed the comparison in drying behaviour between these two positions. After freezedrying, the cake appearance of each vial was visually evaluated to look for signs of macroscopic collapse. Slight shrinkage of the cake was attributed to the amorphous characteristics of the sucrose formulation and was not considered as macroscopic collapse [26].

\section{Results}

\subsection{Experimental determination of the vial heat transfer coefficient}

$K_{v}$ as function of $P_{c}$ is displayed in figure 3 Higher values of $P_{c}$ are associated with a significant increase in $K_{v}$, as reported before [13, 23, 27]. Sub-optimal contact between the stainless steel shelf and the bottom of the glass vials leads to an enhanced conductive heat flux for an increased gas molecule density between both surfaces. Furthermore, $K_{v}$ is higher for vials situated at the edge of the batch due to the significantly higher contribution of radiation energy coming from the surroundings, compared to vials in the center of the batch [13, 23, 27. Therefore, discrimination was made between these two groups of vials. The relation between $K_{v}$ and $P_{c}$ is described by equation 3 . For both groups of vials, non-linear regression was applied to fit equation 3 to the data, resulting in the parameters describing $K_{v}$ as function of $P_{c}$. For the center vials, $\alpha=6.11 \mathrm{~J} /\left(\mathrm{m}^{2} \mathrm{sK}\right)(95 \%$ CI $[5.21,7.01]), \beta=1.54 \mathrm{~J} /\left(\mathrm{m}^{2} \mathrm{sKPa}\right)([1.31,1.78])$ and $\gamma=$ $0.0461 / \mathrm{Pa}([0.037,0.055])$ were obtained. For the edge vials, $\alpha=11.16 \mathrm{~J} /\left(\mathrm{m}^{2} \mathrm{sK}\right)(95 \% \mathrm{CI}[10.16,12.16])$, $\beta=1.44 \mathrm{~J} /\left(\mathrm{m}^{2} \mathrm{sKPa}\right)([1.20,1.69])$ and $\gamma=0.0401 / \mathrm{Pa}([0.031,0.050])$ were obtained. As the edge vials receive additional radiation energy, the risk of cake collapse is higher in these vials compared to the center vials. For this reason, the parameters describing $K_{v}$ as function of $P_{c}$ for the edge vials were selected as input factors for the mechanistic model. Consequently, the predicted drying time will be underestimated 
for the center vials. To assure primary drying is finished for all vials, the total drying time was prolonged by maintaining the final optimal combination of $T_{s}$ and $P_{c}$ for a few additional hours on top of the optimal dynamic trajectory for the edge vials, based on the coefficients describing $K_{v}$ for these center vials (Section 3.4 .

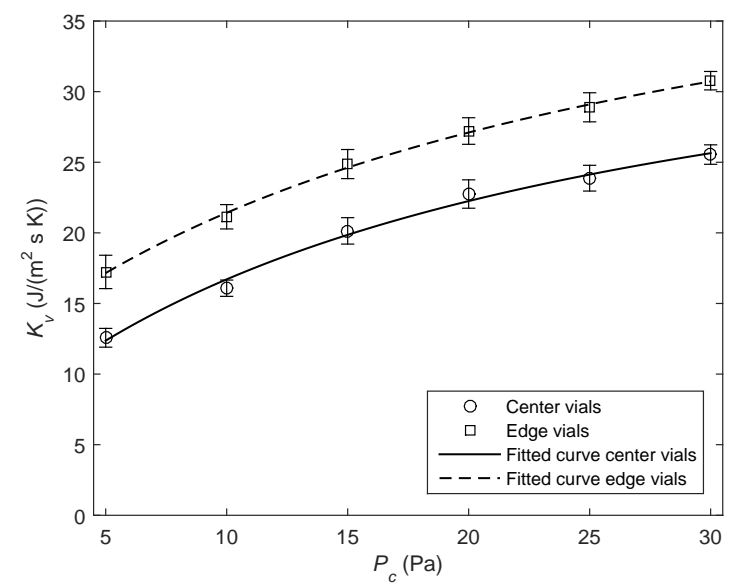

Figure 3: Vial heat transfer coefficient $K_{v}$ plotted as function of chamber pressure $P_{c}$ for edge (squares) and center vials (circles)

\subsection{Dynamic Design Space for Risk of Failure of $1 \%$}

The procedure to determine the optimal combination of $P_{c}$ and $T_{s}$ is presented in figure 4 . The coloured rectangle in the figures represents the grid used at the current time step. The full range of $P_{c}$ is explored, while the range for $T_{s}$ is based on the maximum temperature ramp which can be obtained by the shelves of the freeze-dryer. As the time step is small at the start of the calculations, the range of $T_{s}$ is also small. With the increase of the time step throughout the simulation, the width of the range of $T_{s}$ also increases. On the left side of the figure, the calculated sublimation rate $\dot{m}_{s u b}$ is given for each combination of $P_{c}$ and $T_{s}$ in the explored range. In the figure in the center, the product temperature $T_{i}$ is plotted, while the black line indicates $T_{i, c r i t}$. Below this black line the critical value is not exceeded. The right part of figure 4 indicates for which $P_{c}-T_{s}$-combinations the choked flow criterion is exceeded, both at vial and dryer-level, which is never the case here. After determining the optimal combination of $T_{s}$ and $P_{c}$, which is indicated with a black dot in the left figure, the uncertainty level on $L_{\text {dried }}$ is calculated using an uncertainty analysis.

The evolution of $L_{\text {dried }}$ for an RoF of $1 \%$ is given in figure 5 . Initially, $P_{c}$ is equal to the ambient pressure, the condition used during the freezing step. At this high pressure, the sublimation rate is zero and $L_{d r i e d}$ remains at zero, until $P_{c}$ becomes lower than the ice vapour pressure and sublimation starts. Later on, during primary drying, the increase in $L_{\text {dried }}$ is almost linear.

In figure 6, the optimal values for $T_{s}$ and $P_{c}$ are presented. Initially, $T_{s}$ equals $-45^{\circ} \mathrm{C}$, the temperature used during the freezing step. As soon as sublimation starts, the optimal value of $T_{s}$ increases at the maximum rate which can be obtained by the freeze-dryer. After approximately $1 \mathrm{~h}$, the optimal value starts to decrease again due to the limitation of $T_{i, c r i t} . P_{c}$ is lowered at the start of primary drying and, after reaching vacuum conditions, the optimal value is hardly ever changing.

In figure 7 the uncertainty level on $L_{\text {dried }}\left(S_{L_{\text {dried }}}\right)$ is plotted in function of $L_{d r i e d}$. The increase of the uncertainty on $L_{\text {dried }}$ with the drying time is obvious, i.e. the error is based on deviations in the previous steps, which leads to a cumulative effect: error propagation.

Figure 8 presents the uncertainty level on $T_{s}$ which is asymmetric. When the $T_{s}$ is increasing, i.e. the first $1 \mathrm{~h}$, the actual value runs behind due to thermal inertia of the shelves, and therefore, the uncertainty is higher at the lower boundary compared to the upper boundary. The opposite is true when the temperature 


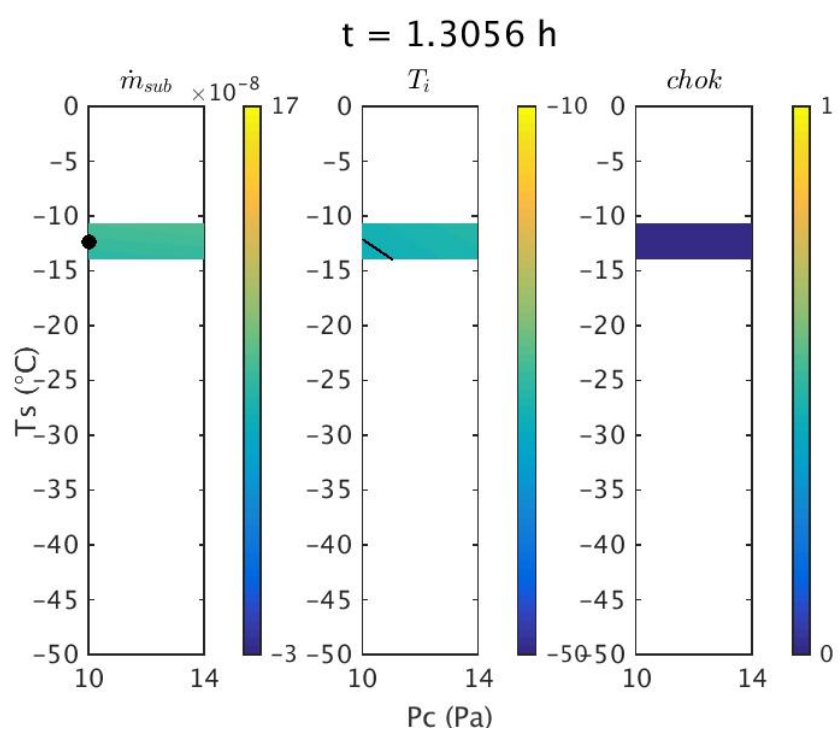

Figure 4: Determination of the optimal values for $P_{c}$ and $T_{s}$ (e.g., at $t=1.31 \mathrm{~h}$ ): In the left figure, $\dot{m}_{\text {sub }}$ is plotted for each combination of $P_{c}$ and $T_{s}$ where the optimal $T_{s} P_{c}$ combination is indicated with a black dot. In the middle figure, $T_{i}$ is plotted for each combination of $P_{c}$ and $T_{s}$ with the black line indicating $T_{i, c r i t}$; below this line $T_{i, c r i t}$ is not exceeded. In the right figure, the choked flow criterion is evaluated: the result is either 0 (not exceeding the limit) or 1 (exceeding the limit).

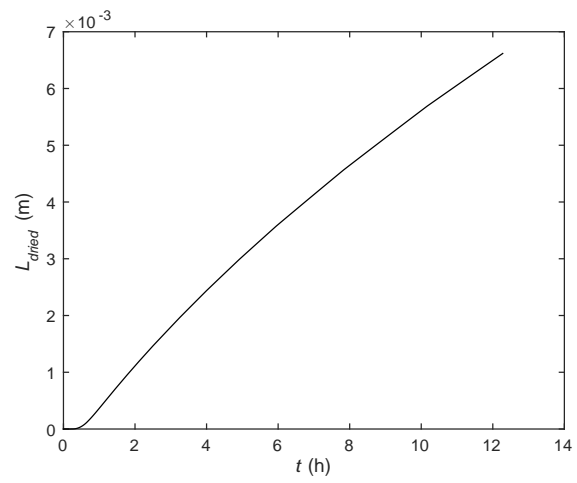

Figure 5: Evolution of $L_{\text {dried }}$ for an RoF acceptance level of $1 \%$

starts to decrease, i.e. after $1 \mathrm{~h}$.

\subsection{Comparison of dynamic Design Space for different Risk of Failure levels}

In the left part of figure 9, $T_{s}$ is plotted in function of $t$ for four different RoF acceptance levels. A higher RoF allows the use of less conservative settings, therefore, $T_{s}$ is obviously higher compared to lower RoF levels. The difference in the maximum value of $T_{s}$ is around $17^{\circ} \mathrm{C}$ between an RoF value of $99 \%$ and $50 \%$. Between $1 \%$ and $99 \%$ this difference is about $29^{\circ} \mathrm{C}$. The corresponding evolution of $T_{i}$ is given in the right part of figure 9. It is obvious that $T_{i}$ is higher for higher RoF levels. When the RoF value is higher than $50 \%$, the chance that $T_{i, c r i t}$ is exceeded is higher than $50 \%$. Therefore, $T_{i, c r i t}$ is evolving after the start-up phase towards the nominal value of the $T_{i}$, i.e. $-32.5^{\circ}$ when the RoF is $50 \%$. For an RoF of $99 \%, T_{i}$ is evolving towards $-30^{\circ}$, which is higher than $T_{i, \text { crit }}$. For an RoF of $1 \%$ and $25 \%, T_{i}$ is evolving to 

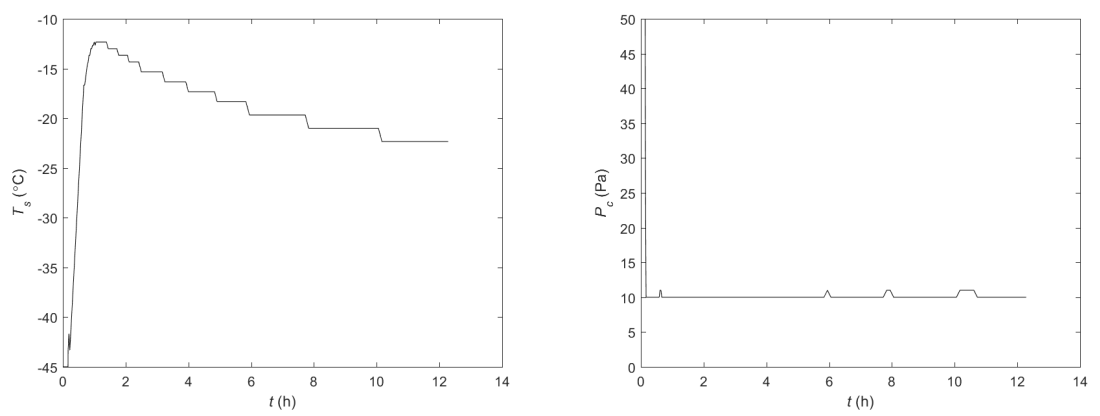

Figure 6: Optimal values for $T_{s}$ (Left) and $P_{c}$ (Right) for an RoF acceptance level of $1 \%$

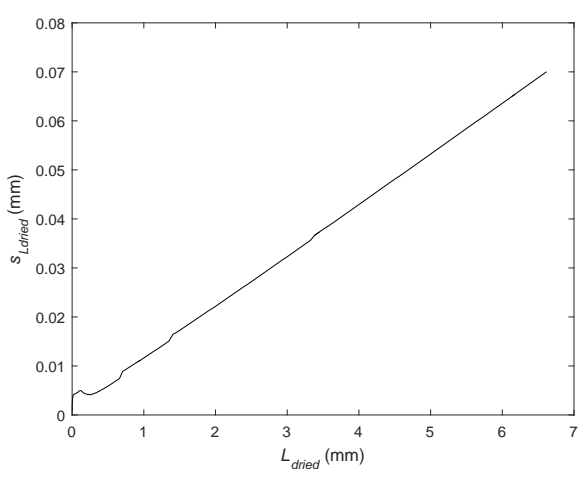

Figure 7: Uncertainty level on $L_{\text {dried }}\left(S_{L_{\text {dried }}}\right)$ for an RoF acceptance level of $1 \%$

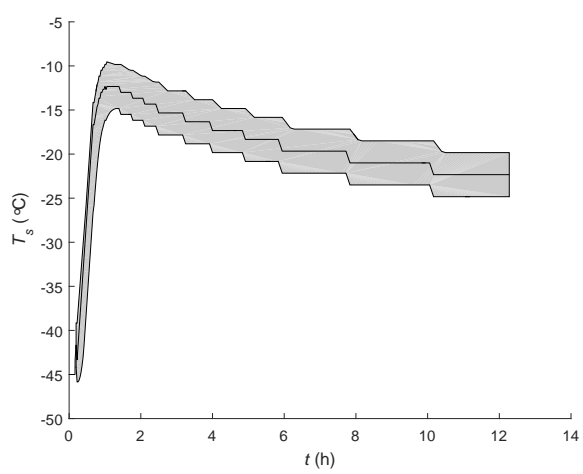

Figure 8: Uncertainty level on $T_{s}$ for an $\mathrm{RoF}$ acceptance level of $1 \%$ for the model formulation

respectively $-34.5^{\circ}$ and $-33^{\circ}$, both lower than $T_{i, \text { crit }}$.

The difference in $P_{c}$ for different values of RoF is negligible (Figure 10. At the start, $P_{c}$ is decreasing to vacuum conditions, while an increase is noted after around $0.6 \mathrm{~h}$. This increase is higher for a higher RoF level. It is clear that the total value of $t$ is larger when the RoF is lower. The computed drying time for the edge vials is $12.28 \mathrm{~h}, 9.97 \mathrm{~h}, 9.05 \mathrm{~h}$ and $6.67 \mathrm{~h}$ for an RoF of $1 \%, 25 \%, 50 \%$ and $99 \%$, respectively. It has to be remarked that these drying times are computed based on the coefficients describing $K_{v}$ for the edge vials. As compensation, the total drying time for the complete batch is prolonged by maintaining the final optimal combination of $T_{s}$ and $P_{c}$ for a few additional hours on top of the optimal dynamic trajectory for the edge vials as calculated based on the coefficients describing $K_{v}$ for the center vials (Section 3.4). 

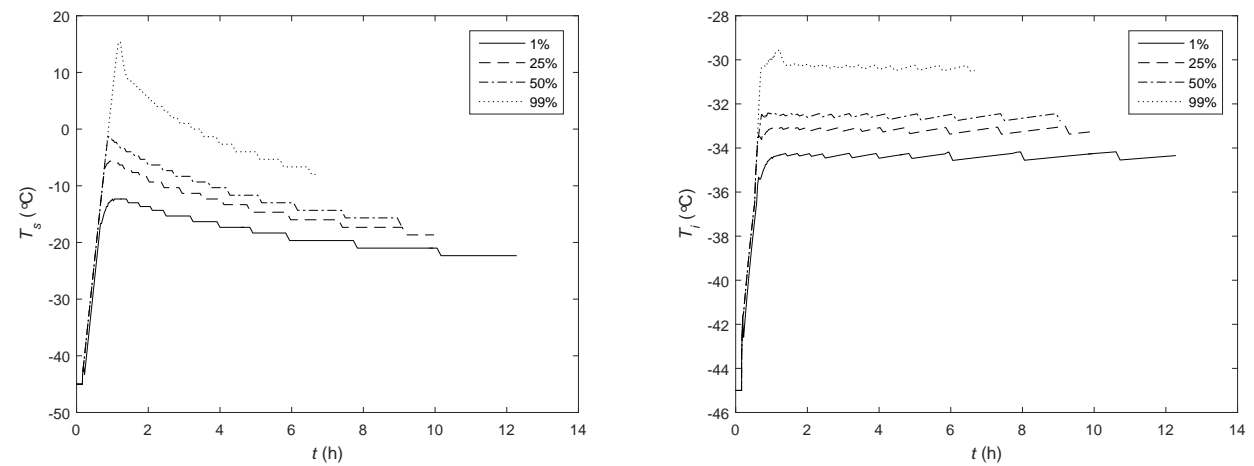

Figure 9: Optimal values for $T_{s}$ and the evolution of $T_{i}$ for an $\mathrm{RoF}$ acceptance level of $1 \%, 25 \%, 50 \%$ and $99 \%$

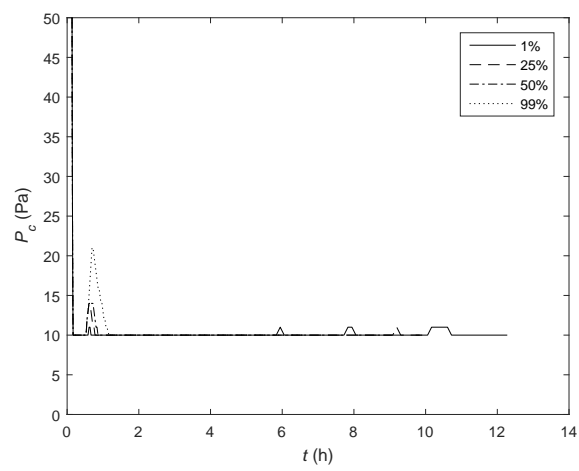

Figure 10: Optimal values for $P_{c}$ for an RoF acceptance level of $1 \%, 25 \%, 50 \%$ and $99 \%$

\subsection{Experimental verification of the dynamic Design Space}

In figure 11, the monitored $T_{i}$ is plotted in function of $t$ for an RoF acceptance level of $1 \%, 25 \%, 50 \%$ and $99 \%$, while the horizontal line represents $T_{i, \text { crit }}$. Immediately after introducing the vacuum, $T_{i}$ was very low (below $-40^{\circ} \mathrm{C}$ ). With the progress of drying, $L_{\text {dried }}$ and $R_{p}$ simultaneously started to increase. Consequently, the mass transfer of water vapour through the dried layer got more obstructed, associated with a local pressure rise at the sublimation front and, hence, an increase in $T_{i}$, eventually leading to a gradual limitation of $T_{s}$. $T_{i}$ was measured at the bottom of the vial, while there is a (small) temperature gradient across the frozen product layer between the sublimation front and the bottom of the vial. In general, the temperature measured at the bottom of the vial is slightly higher compared to the sublimation front. As the sublimation interface gradually moved downwards during primary drying, this temperature gradient became smaller, which might explain the gradual decrease in $T_{i}$. This observed decrease is mostly relevant for the more aggressive cycles with a higher RoF Therefore, this feature might be linked to the occurence of collapse at the ice-dried product interface due to the exceedance of $T_{i, c r i t}$. The increased pore size in the dried product layer might reduce $R_{p}$ and in turn $T_{i}$ [28, 29]. Near the end of primary drying, when the ice in contact with the thermocouple was completely sublimated, $T_{i}$ increased drastically, as more energy was transferred to the vial than was removed via ice sublimation.

The measured value of $T_{i}$ was obviously higher with the increase in RoF acceptance level (Figure 11), as $T_{s}$ was set at a higher (mean) value for each corresponding verification run (Figure 9p. $T_{i}$ was also higher for edge vials compared to center vials during the same $\mathrm{RoF}$ verification run, due to the additional radiation energy originating from the surroundings received by the edge vials (Section 3.1). During the experimental verification run based on an RoF acceptance level of $50 \%$ and $99 \%, T_{i}$ exceeds $T_{i, c r i t}$ in both the edge and center vials. For an $\mathrm{RoF}$ of $25 \%, T_{i}$ remains below $T_{i, \text { crit }}$ in the center vials, while $T_{i}$ is slightly above $T_{i, \text { crit }}$ 

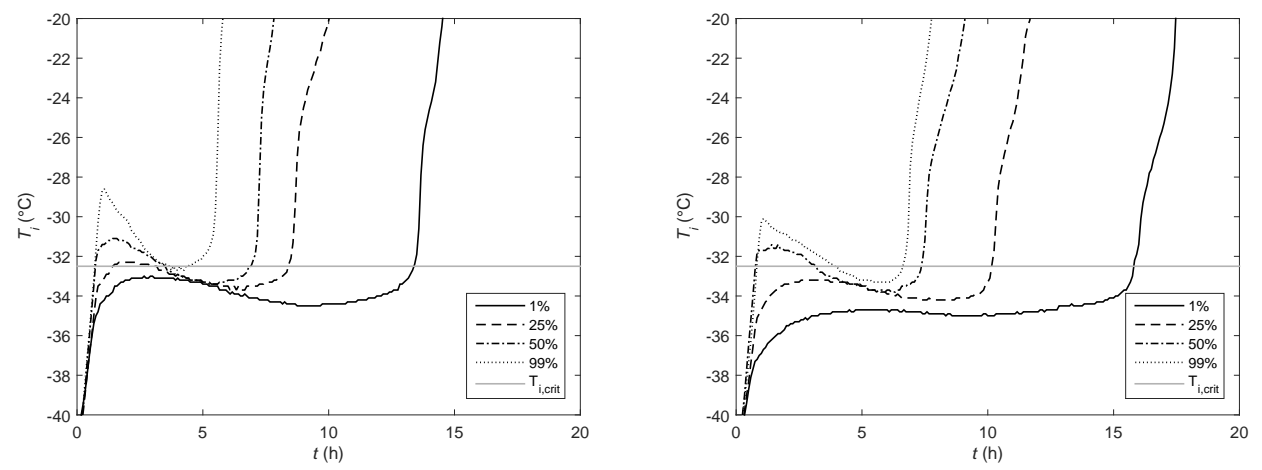

Figure 11: Experimental $T_{i}$ for an $\mathrm{RoF}$ acceptance level of $1 \%, 25 \%, 50 \%$ and $99 \%$ and $T_{i, \text { crit }}$ for an edge (left) and center (right) vial

in the edge vials. For an $\mathrm{RoF}$ of $1 \%, T_{i}$ remains below $T_{i, \text { crit }}$ in both the edge and center vials. These temperature data can be related to the resulting cake appearance after each verification run. Macroscopic cake collapse was observed in a significant part of the batch for an RoF acceptance level of $50 \%$ and $99 \%$, as $T_{i, c r i t}$ was greatly exceeded during primary drying (Figure 12). The degree of collapse was more severe for a higher RoF. The majority of the vials where loss of structure occurred, was situated at the edge of the batch, due to the additional energy transfer (Section 3.1). The vials obtained during the verification run with an $\mathrm{RoF}$ of $25 \%$ did not show any sign of (macroscopic) collapse. Despite (just) exceeding $T_{i, \text { crit }}$ in the edge vials, $T_{i}$ remained below $T_{c}$. $T_{i, c r i t}$ was assumed to be equal to $T_{g}^{\prime}$ of the model formulation (Section 2.2. Because the viscosity near $T_{g}^{\prime}$ is sufficiently high to prevent viscous flow, no macroscopic collapse was observed. $T_{i, \text { crit }}$ was never exceeded during the verification run for an RoF of $1 \%$. Logically, an acceptable cake appearance was obtained at the end of this run (Figure 12). Due to the amorphous characteristics of the product, partial cake shrinkage of approximately $10 \%$ was observed for all non-collapsed vials in the batch.

For each RoF acceptance level, the computed primary drying endpoint with a certainty of $99 \%$, based on
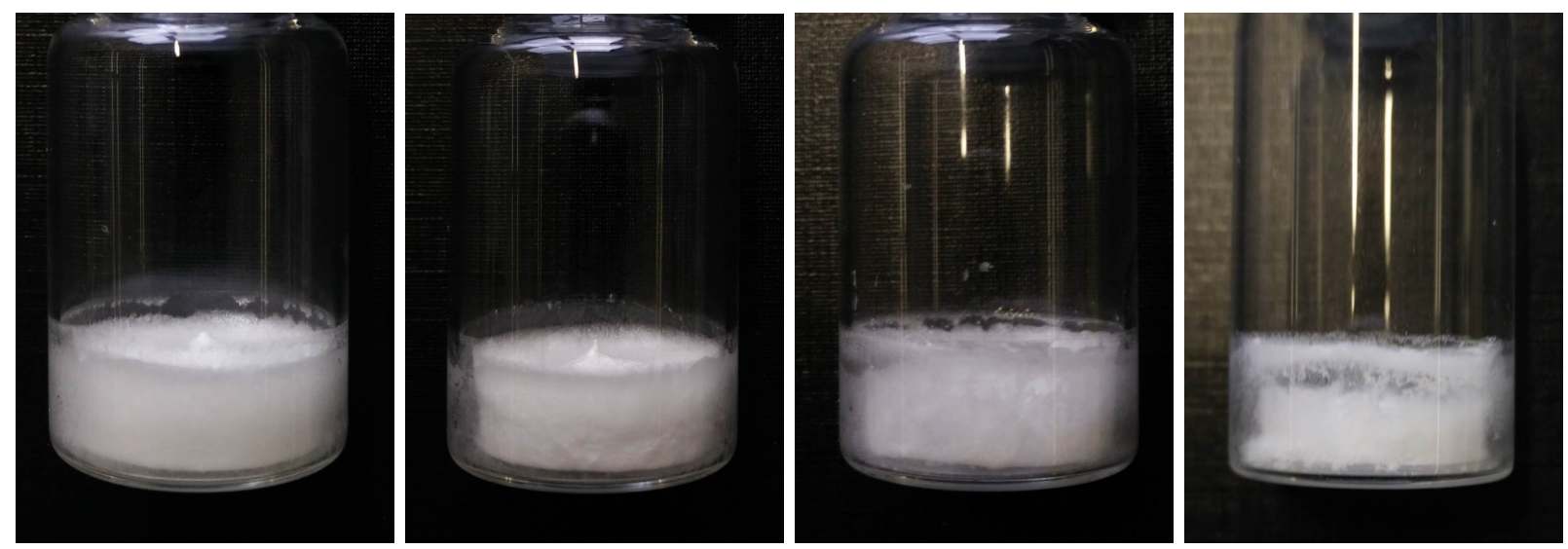

Figure 12: Cakes yielded after experimental verification runs for RoF of $1 \%$ (left), 25\% (middle left), $50 \%$ (middle right) and $99 \%$ (right)

the coefficients describing $K_{v}$ for the center vials, is listed in table 3 These predictions are in accordance with the experimentally determined primary drying endpoint, determined via the ratio of the relative and absolute pressure measurement. For each $\mathrm{RoF}$ acceptance level, the experimental endpoint was situated below the computed limit, indicating that primary drying was finished before the shelf temperature ramp 
to secondary drying was started.

Table 3: The experimentally determined primary drying time for the center vials for different RoF acceptance levels compared with the computed primary drying endpoint with $99 \%$ certainty

\begin{tabular}{c|c|c} 
Risk of Failure & Computed primary drying endpoint $(\mathrm{h})$ & Experimental value $(\mathrm{h})$ \\
\hline $1 \%$ & 17.61 & 16.83 \\
$25 \%$ & 14.19 & 13.59 \\
$50 \%$ & 12.61 & 11.72 \\
$99 \%$ & 9.22 & 9.09
\end{tabular}

\section{Discussion}

Conventionally, the primary drying step of a freeze-drying process is characterized by fixed settings for the adaptable process parameters $T_{s}$ and $P_{c}$. These suboptimal process conditions lead to the prolongation of the primary drying time, associated with an increase in production costs and inefficient usage of the equipment. Alternatively, mechanistic modelling is a cost-effective and efficient approach for the development and optimization of freeze-drying cycles, via the construction of the Design Space for the primary drying step. Because some model input variables change with the primary drying progress, e.g. the gradual increase in $L_{\text {dried }}$ corresponds to an increase in $R_{p}$ (Section 1), the optimal combination of $T_{s}$ and $P_{c}$ also changes throughout the process. Instead of a static Design Space, this leads to the construction of a dynamic Design Space, associated with an increased processing efficiency. Because model input variables and process parameters are often an estimation of the real value and due to the fact that several assumptions and resulting simplifications were included in the model, an uncertainty analysis was conducted to quantitatively estimate the risk of cake collapse for each combination of the adaptable process parameters $T_{s}$ and $P_{c}$ in the dynamic Design Space. In addition, experimental verification of the Design Space is required before conclusions can be drawn with the final aim of implementing the Design Space in production processes.

The experimental run based on the nominal values of the input parameters (i.e. RoF of 50\%) led to cake collapse in a significant number of vials, mainly situated at the edge of the batch, which would in practice lead to rejection of the complete batch. This observation indicates the necessity of including the parameter uncertainty during Design Space construction via the uncertainty analysis. The nominal values of the input variables are often associated with a certain degree of uncertainty. For instance, $R_{p}$ is characterized by one global set of coefficients describing $R_{p}$ in function of $L_{\text {dried }}$. However, this parameter is inherently associated with a high inter-vial variability due to the stochastic nature of ice nucleation leading to differences in ice crystal size, which is linked to the final pore size in the dried product [3. As the pore size impacts the mass flow dynamics through the dried layer, $R_{p}$ is subject to an immense vial-to-vial variability [30. The inclusion of an annealing step improves the inter-vial homogeneity in pore size [4. However, an assessment of the uncertainty on $R_{p}$ is still required, as indicated during the experimental verification of the dynamic Design Space.

The observation that cake collapse mainly occurred in the vials situated at the edge of the batch was attributed to the additional radiation energy transfer to these vials (Section 3.1). As $K_{v}$ is highly different between the edge and center vials [13, 23, 27, which is inherent to the design of the freeze-dryer, a global value for the quantification of $K_{v}$ for the whole batch would be associated with a very high uncertainty for each individual vial. This issue was addressed by dividing the batch in two groups, based on whether radiation from the surroundings contributed to the energy transfer or not (Section 2.3p. This way, the uncertainty on $K_{v}$ was reduced as within each individual group the variability was much smaller. However, due to local differences in energy transfer to the individual vials of each group depending on the location of the vial in the drying chamber, the shape of the vial bottom and temperature differences across the shelves, $K_{v}$ should still be included in the uncertainty analysis. In addition, the inherent inter-vial variability in both $R_{p}$ and $K_{v}$ also yields a potential increased variability in final product quality, which however is difficult to avoid due to the design of the equipment. 
As the total energy transfer to the edge vials is significantly higher compared to the center vials, this group was considered for the construction of the dynamic Design Space, while the center vials were the limiting factor regarding the computation of the primary drying endpoint. The endpoint of primary drying was experimentally verified based on the pressure ratio measurement between a Pirani gauge and a capacitance manometer. To avoid the introduction of secondary drying before primary drying is finished, the one-sided 97.5\% confidence limit is considered as the most relevant and safe parameter in estimating the primary drying endpoint. An even better approach, which would fit the Process Analytical Technology (PAT) initiative issued by the Food and Drug Administration (FDA), would be to monitor the pressure ratio realtime and in-line and to start the secondary drying ramp-up when a predefined threshold value is reached 31 .

The parameters which should be included in the uncertainty analysis and their respective uncertainty level should be considered very carefully, as they impact the Design Space [13. Preferably, the uncertainty level is based on experimental data, e.g. $T_{s}, K_{v}, r_{v, i}$ or $r_{v, o}$. In case experiments do not give an indication about the degree of uncertainty, the uncertainty level should be estimated based on expert knowledge. This estimation should be conservative, associated with rather broad uncertainty levels, otherwise this could lead to collapse of the product, even for very low risk of failure acceptance levels.

\section{General conclusion}

The dynamic Design Space for the primary drying step of the freeze-drying process was constructed, allowing the determination of the optimal combination of the adaptable process parameters $T_{s}$ and $P_{c}$ in function of the primary drying time. The inclusion of the uncertainty analysis extended with the error propagation, lead to the quantification and control of the risk of cake collapse and rejection of the batch. The dynamic Design Space was verified with experimental data for different risk of failure acceptance levels. The experimental run based on the nominal values of the input variables (i.e. RoF of 50\%) resulted in cake collapse for a significant part of the batch. An acceptable cake appearance was only obtained for a minimum risk of failure acceptance level of $25 \%$. This study demonstrates that the uncertainty analysis is an essential step in the establishment of the dynamic Design Space, with a controlled risk of cake collapse.

\section{Acknowledgment}

Financial support for this research from the Fund for Scientific Research Flanders (FWO Flanders Ph.D. fellowship Séverine Thérèse F.C. Mortier) is gratefully acknowledged.

\section{Nomenclature}

$\alpha \quad$ Constant describing $K_{v}$ in function of $P_{c}\left(\mathrm{~J} /\left(\mathrm{m}^{2} \mathrm{sK}\right)\right)$

$\beta \quad$ Constant describing $K_{v}$ in function of $P_{c}\left(\mathrm{~J} /\left(\mathrm{m}^{2} \mathrm{sKPa}\right)\right)$

$\Delta H_{\text {sub }}$ Latent sublimation heat of ice $(\mathrm{J} / \mathrm{mol})$

$\Delta m \quad$ Mass of sublimated ice $(\mathrm{kg})$

$\Delta t \quad$ Primary drying time interval (s)

$\dot{m}_{\text {sub,chok,dryer }}$ Maximum sublimation rate avoiding choked flow at dryer level $(\mathrm{kg} / \mathrm{s})$

$\dot{m}_{\text {sub,chok,vial }}$ Maximum sublimation rate avoiding choked flow at vial level $(\mathrm{kg} / \mathrm{s})$

$\dot{m}_{\text {sub }}$ Sublimation rate $(\mathrm{kg} / \mathrm{s})$

$\epsilon \quad$ Volume fraction of ice (-) 
$\gamma \quad$ Constant describing $K_{v}$ in function of $P_{c}(1 / \mathrm{Pa})$

$\rho_{\text {ice }} \quad$ Density of ice $\left(\mathrm{kg} / \mathrm{m}^{3}\right)$

$A_{p} \quad$ Product area available for sublimation $\left(\mathrm{m}^{2}\right)$

$A_{v} \quad$ Outer vial area $\left(\mathrm{m}^{2}\right)$

$A_{R_{p}} \quad$ Constant describing $R_{p}$ in function of $l(1 / \mathrm{s})$

$B_{R_{p}} \quad$ Constant describing $R_{p}$ in function of $l(1 / \mathrm{m})$

$k \quad$ Ratio of specific heats (-)

$K_{v} \quad$ Vial heat transfer coefficient $\left(\mathrm{J} /\left(\mathrm{m}^{2} \mathrm{sK}\right)\right)$

$L_{\text {dried }}$ Dried layer thickness $(\mathrm{m})$

$M \quad$ Molecular weight $(\mathrm{kg} / \mathrm{mol})$

$n_{\text {vials }}$ Amount of vials (-)

$P_{c} \quad$ Chamber pressure $(\mathrm{Pa})$

$P_{w, c} \quad$ Partial water pressure in the drying chamber $(\mathrm{Pa})$

$P_{w, i} \quad$ Vapour pressure at the sublimation interface $(\mathrm{Pa})$

$R \quad$ Gas constant $(8.3144621 \mathrm{~J} /(\mathrm{K} \mathrm{mol}))$

$R_{p} \quad$ Dried product mass transfer resistance $(\mathrm{m} / \mathrm{s})$

$r_{d} \quad$ Equivalent radius corresponding to duct opening between chamber and condenser $(\mathrm{m})$

$R_{p, 0} \quad$ Constant describing $R_{p}$ in function of $l(\mathrm{~m} / \mathrm{s})$

$r_{v, i} \quad$ Inner radius of the glass vial $(\mathrm{m})$

$r_{v, n} \quad$ Equivalent radius corresponding to vial neck opening $(\mathrm{m})$

$r_{v, o} \quad$ Outer radius of the glass vial $(\mathrm{m})$

$S_{L_{\text {dried }}}$ Uncertainty level on $L_{\text {dried }}(\mathrm{m})$

$t \quad$ Primary drying time (h)

$T_{b} \quad$ Ice temperature at bottom of the vial $(\mathrm{K})$

$T_{c} \quad$ Collapse temperature $(\mathrm{K})$

$T_{e} \quad$ Eutectic temperature $(\mathrm{K})$

$T_{g} \quad$ Glass transition temperature of the freeze-dried product $(\mathrm{K})$

$T_{g}^{\prime} \quad$ Glass transition temperature of the maximum freeze-concentrated formulation $(\mathrm{K})$

$T_{i} \quad$ Product temperature at sublimation front $(\mathrm{K})$

$T_{r} \quad$ Temperature of the water vapour $(\mathrm{K})$

$T_{s} \quad$ Shelf fluid inlet temperature $(\mathrm{K})$

$T_{i, c r i t}$ Critical product temperature $(\mathrm{K})$

$V \quad$ Filling volume $(\mathrm{mL})$ 


\section{References}

[1] M. J. Pikal, Freeze drying, in: Encyclopedia of pharmaceutical technology, 2002, pp. 1299-1326.

[2] P.-J. Van Bockstal, L. De Meyer, J. Corver, C. Vervaet, T. De Beer, Non-contact infrared-mediated heat transfer during continuous freeze-drying of unit doses, J. Pharm. Sci. 106 (2017) 71-82.

[3] J. Kasper, W. Friess, The freezing step in lyophilization: physico-chemical fundamentals, freezing methods and consequences on process performance and quality attributes of biopharmaceuticals, Eur. J. Pharm. Biopharm. 78 (2011) $248-263$.

[4] J. A. Searles, J. F. Carpenter, T. W. Randolph, Annealing to optimize the primary drying rate, reduce freezing-induced drying rate heterogeneity, and determine Tg' in pharmaceutical lyophilization, J. Pharm. Sci. 90 (7) (2001) 872-887.

[5] A. Hawe, W. Friess, Impact of freezing procedure and annealing on the physico-chemical properties and the formation of mannitol hydrate in mannitol-sucrose-NaCl formulations, Eur. J. Pharm. Biopharm. 64 (3) (2006) $316-325$.

[6] X. Lu, M. J. Pikal, Freeze-drying of mannitol-trehalose-sodium chloride-based formulations: the impact of annealing on dry layer resistance to mass transfer and cake structure, Pharm. Dev. Technol. 9 (1) (2004) 85-95.

[7] L. X. Yu, Pharmaceutical quality by design: Product and process development, understanding, and control, Pharm. Dev. Technol. 25 (2008) 781-791.

[8] D. Fissore, R. Pisano, A. A. Barresi, Monitoring of the Secondary Drying in Freeze-Drying, J. Pharm. Sci. 100 (2011) $732-742$.

[9] R. Pisano, D. Fissore, A. A. Barresi, R. Pisano, D. Fissore, A. A. Barresi, Quality by Design in the Secondary Drying Step of a Freeze-Drying Process, Dry. Technol. 30 (2012) 1307-1316.

[10] A. Giordano, A. A. Barresi, D. Fissore, On the use of mathematical models to build the design space for the primary drying phase of a pharmaceutical lyophilization process., J. Pharm. Sci. 100 (2011) 311-324.

[11] L. N. Mockus, T. W. Paul, N. A. Pease, N. J. Harper, P. K. Basu, E. A. Oslos, G. A. Sacha, W. Y. Kuu, L. M. Hardwick, J. J. Karty, M. J. Pikal, E. Hee, M. A. Khan, S. L. Nail, Quality by design in formulation and process development for a freeze-dried, small molecule parenteral product: a case study, Pharm. Dev. Technol. 16 (2011) 549-576.

[12] D. Fissore, R. Pisano, A. A. Barresi, Advanced approach to build the design space for the primary drying of a pharmaceutical freeze-drying process, J. Pharm. Sci. 100 (2011) 4922-4933.

[13] S. Mortier, P. J. Van Bockstal, J. Corver, I. Nopens, K. Gernaey, T. De Beer, Uncertainty analysis as essential step in the establishment of the dynamic Design Space of primary drying during freeze-drying, Eur. J. Pharm. Biopharm. 103 (2016) 71-83.

[14] V. R. Koganti, E. Y. Shalaev, M. R. Berry, T. Osterberg, M. Youssef, D. N. Hiebert, F. A. Kanka, M. Nolan, R. Barrett, G. Scalzo, G. Fitzpatrick, N. Fitzgibbon, S. Luthra, L. Zhang, Investigation of design space for freeze-drying: use of modeling for primary drying segment of a freeze-drying cycle., AAPS PharmSciTech 12 (2011) 854-861.

[15] S. L. Nail, J. A. Searles, Elements of Quality by Design in development and scale-up of freeze-dried parenterals, BioPharm Int. 21 (2008) 44-52.

[16] L. Hardwick, C. Paunicka, M. Akers, Critical Factors in the Design and Optimisation of Lyophilisation Processes, IPT 26 (2008) 70-74.

[17] S. M. Patel, S. Chaudhuri, M. J. Pikal, Choked flow and importance of Mach I in freeze-drying process design, Chem. Eng. Sci. 65 (2010) 5716-5727.

[18] S. Bosca, D. Fissore, M. Demichela, Risk-Based Design of a Freeze-Drying Cycle for Pharmaceuticals, Ind. Eng. Chem. Res. 54 (2015) 12928-12936.

[19] R. Bogner, M. J. Pikal, The Incredible Shrinking Design Space: Using Risk Tolerance to Define Design Space for Primary Drying, Notes of Freeze Drying of Pharmaceuticals and Biologicals Conference, Garmisch-Partenkirchen (2010) $184-198$.

[20] W. Y. Kuu, L. M. Hardwick, M. J. Akers, Rapid determination of dry layer mass transfer resistance for various pharmaceutical formulations during primary drying using product temperature profiles, Int. J. Pharm. 313 (2006) 99-113.

[21] F. Graziani, Uncertainty and Sensitivity Analysis for Models of Complex Systems, Springe, Berlin, 2008.

[22] I. M. Sobol', On quasi-Monte Carlo integrations, Math. Comput. Simulat. 47 (1998) $103-112$.

[23] R. Pisano, D. Fissore, A. A. Barresi, P. Brayard, P. Chouvenc, B. Woinet, Quality by design: optimization of a freezedrying cycle via design space in case of heterogeneous drying behavior and influence of the freezing protocol, Pharm. Dev. Technol. 18 (2013) 280-295.

[24] S. Nail, S. Tchessalov, E. Shalaev, A. Ganguly, E. Renzi, F. Dimarco, L. Wegiel, S. Ferris, W. Kessler, M. Pikal, G. Sacha, A. Alexeenko, T. N. Thompson, C. Reiter, J. Searles, P. Coiteux, Recommended Best Practices for Process Monitoring Instrumentation in Pharmaceutical Freeze Drying2017, AAPS PharmSciTechdoi:10.1208/s12249-017-0733-1

[25] S. M. Patel, T. Doen, M. J. Pikal, Determination of end point of primary drying in freeze-drying process control., AAPS PharmSciTech 11 (1) (2010) 73-84.

[26] S. Rambhatla, J.-P. Obert, S. Luthra, C. Bhugra, M. J. Pikal, Cake shrinkage during freeze drying: a combined experimental and theoretical study., Pharm. Dev. Technol. 10 (2005) 33-40.

[27] S. Rambhatla, M. J. Pikal, Heat and mass transfer scale-up issues during freeze-drying, I: atypical radiation and the edge vial effect., AAPS PharmSciTech 4 (2003) 22-31.

[28] A. Konstantinidis, W. Kuu, L. Otten, S. Nail, R. Sever, Controlled Nucleation in Freeze-drying: Effects on Pore Size in the Dried Product Layer, Mass Transfer Resistance, and Primary Drying Rate, J. Pharm. Sci. 100 (2011) $3453-3470$.

[29] W. Kuu, K. O'Brien, L. Hardwick, T. Paul, Product mass transfer resistance directly determined during freeze-drying cycle runs using tunable diode laser absorption spectroscopy (TDLAS) and pore diffusion model, Pharm. Dev. Technol. 16 (2011) 343-357. 
[30] S. Rambhatla, R. Ramot, C. Bhugra, M. J. Pikal, Heat and mass transfer scale-up issues during freeze drying: II. Control and characterization of the degree of supercooling., AAPS PharmSciTech 5 (2004) 54-62.

[31] Food and Drug Administration, Guidance for Industry PAT: A Framework for Innovative Pharmaceutical Development, Manufacuring, and Quality Assurance (2004).

URL https://www.fda.gov/downloads/Drugs/GuidanceComplianceRegulatoryInformation/Guidances/ucm070305.pdf 